\title{
Measurement of fiducial cross sections of the 125 GeV Higgs boson using the CMS detector
}

\author{
Andre Sznajder for the CMS Collaboration* \\ State University of Rio de Janeiro(UERJ) \\ E-mail: Andre.Sznajder@cern.ch
}

\begin{abstract}
The status of the fiducial cross sections of the $125 \mathrm{GeV}$ Higgs boson using the CMS detector are reported. New run-II results at $13 \mathrm{TeV}$ are presented together with the run-I measurements $7 \mathrm{TeV}$ and $8 \mathrm{TeV}$. Differential and integrated fiducial cross sections for Higgs production in the decay channels $H \rightarrow Z Z \rightarrow 4 l, H \rightarrow \gamma \gamma$ and $H \rightarrow W W \rightarrow 2 l 2 v$ are measured. The differential distributions are measured as a function of the kinematic properties of the final state of the given decay channel. Measurements are found to agree, within experimental uncertainties, with theoretical calculations based on the standard model.
\end{abstract}

38th International Conference on High Energy Physics 3-10 August 2016

Chicago, USA

${ }^{*}$ Speaker. 


\section{Introduction}

In this presentation the status of the differential and integrated fiducial cross sections of the $125 \mathrm{GeV}$ Higgs boson using the CMS detector are reported for the decay channels $H \rightarrow Z Z \rightarrow 4 l$, $H \rightarrow \gamma \gamma$ and $H \rightarrow W W \rightarrow 2 l 2 v$. New run-II results at $13 \mathrm{TeV}$ are presented together with the run-I measurements $7 \mathrm{TeV}$ and $8 \mathrm{TeV}$.

Fiducial measurements are performed in a phase space closely matching detector acceptance, allowing more precise measurements by the elimination of acceptance corrections and minimization of Monte Carlo(model) dependency. Measurement structure for the observables is based on the definition of a fiducial region at generator level to match analysis acceptance. The signal is extracted for each bin of the observable based on the final state of the decay channel and then differential measurement is unfolded from reconstruction to generator level quantities.

\section{2. $H \rightarrow Z Z \rightarrow 4 l$}

Differential and integrated fiducial cross sections for the production of four leptons via the $H \rightarrow Z Z \rightarrow 4 l$ decays $(l=e, \mu)$ are measured in pp collisions at 7, 8 and $13 \mathrm{TeV}$. Measurements are performed with integrated luminosities of $5.1 \mathrm{fb}^{1}, 19.7 \mathrm{fb} b^{1}$ and $2.8 \mathrm{fb} \mathrm{b}^{1}$ respectively.

The fiducial phase space requires at least four leptons (electrons, muons), with at least one lepton having $p_{T}>20 \mathrm{GeV}$, another lepton having $p_{T}>10 \mathrm{GeV}$, and the remaining electrons(muons) having $p_{T}>7(5) \mathrm{GeV}$ respectively. All electrons(muons) must have pseudorapidity $|\eta|<2.5(2.4)$, respectively. In addition, each lepton must satisfy an isolation requirement computed using the $\Sigma p_{T}$ of all stable particles within $\Delta R<0.4$ distance from that lepton.

At least two lepton pairs with same flavor and opposite sign are required and all lepton pairs are used to form $\mathrm{Z}$ boson candidates. The pair with invariant mass closest to the nominal $\mathrm{Z}$ boson mass $(91.188 \mathrm{GeV})$ is taken as the first $\mathrm{Z}$ boson candidate $(Z 1)$, which must satisfy $40<m_{Z 1}<$ $120 \mathrm{GeV}$. The remaining pairs are used to form the candidate $(Z 2)$. In events with more than one $Z 2$ candidate, the pair with the largest sum of the transverse momenta magnitudes, $\Sigma\left|p_{T}\right|$, is chosen and it must satisfy $12<m_{Z 2}<120 \mathrm{GeV}$. Any pair of selected leptons must satisfy $\Delta R_{l_{i} l_{j}}>0.02$. Finally, the invariant mass of the Higgs boson candidate must satisfy $105<m_{4 l}<140 \mathrm{GeV}$. Results are shown in figure Fig.1.

\section{3. $H \rightarrow \gamma \gamma$}

A measurement was carried out of differential cross sections as a function of kinematic observables in the $H \rightarrow \gamma \gamma$ channel, using $19.7 f b^{1}$ of data collected at $8 \mathrm{TeV}$. The signal extraction and the unfolding of experimental resolution were performed simultaneously in all bins of the chosen observables. In this kinematic range, the integrated fiducial cross section was measured to be $32 \pm 10 \mathrm{fb}$.

The fiducial volume of the measurement is defined by two isolated photons in the kinematic range $p_{T}^{\gamma 1} / m_{\gamma \gamma}>1 / 3, p_{T}^{\gamma 2} / m_{\gamma \gamma}>1 / 4$ with photon pseudorapidities within $|\eta|<2.5$. We show the differential cross section distributions in figure Fig. 2 

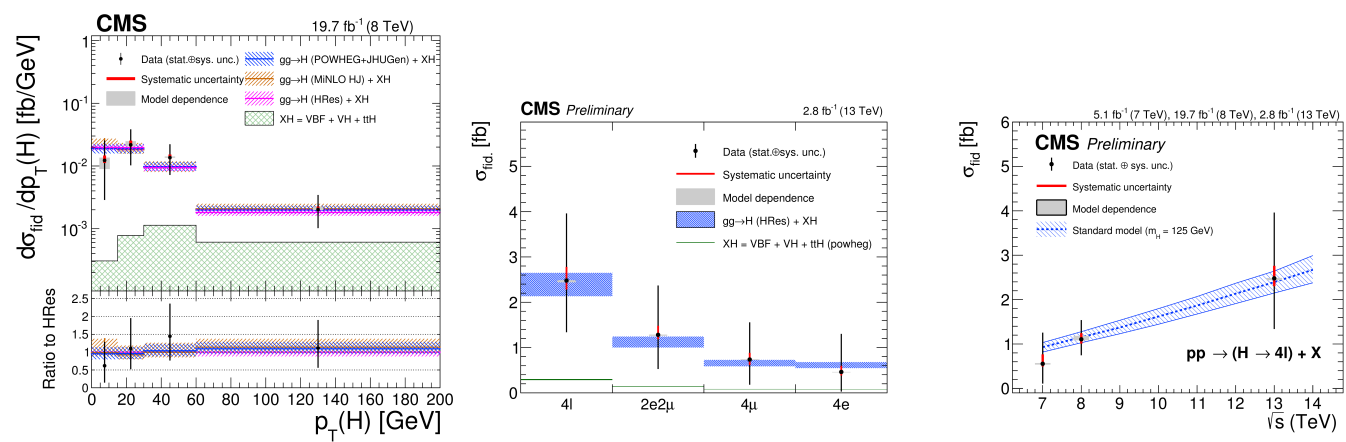

Figure 1: Diferential cross section as a function of the Higgs $p_{T}$ and integrated cross sections per final state and $E_{C M}$
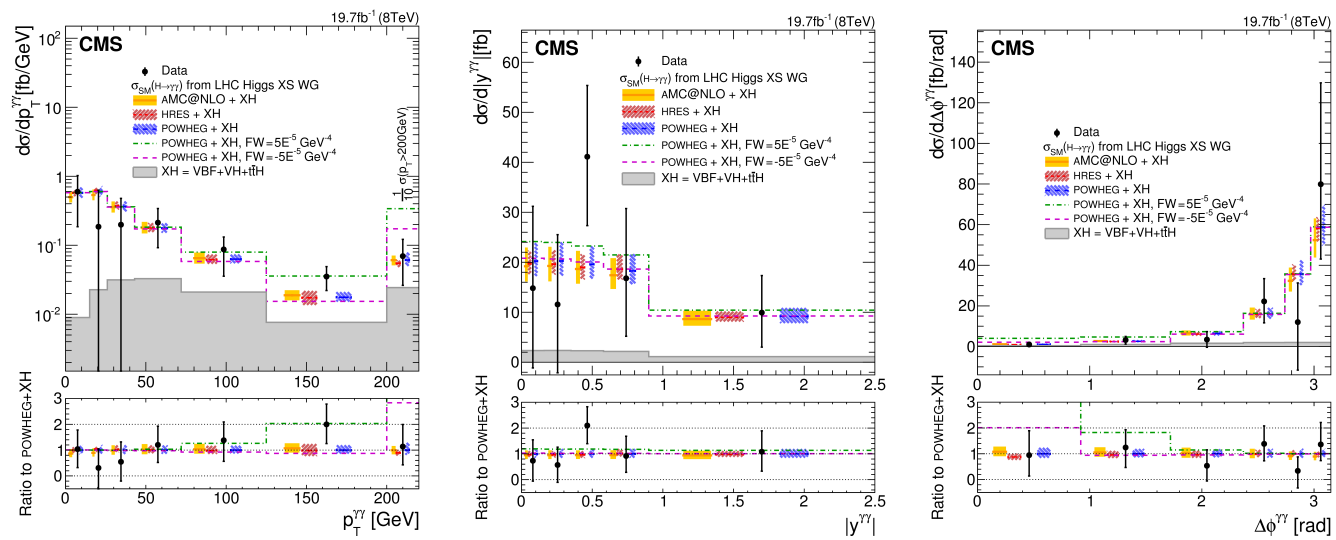

Figure 2: The $H \rightarrow \gamma \gamma$ differential cross sections distributions

4. $H \rightarrow W W \rightarrow 2 l 2 v$

The cross section for Higgs boson production in $p p$ collisions is studied using the $H \rightarrow W W \rightarrow$ $2 l 2 v$ decay mode, where the leptons are oppositely charged electron-muon pairs. The measurements are performed using data collected by the CMS experiment at the LHC at a centre-of-mass energy of $8 \mathrm{TeV}$, corresponding to an integrated luminosity of $19.4 \mathrm{fb}^{1}$. The production cross section times branching fraction in the fiducial phase space is measured to be $39 \pm 8(s t a t) \pm 9(s y s t) f b$. The differential cross section times branching fraction is measured as a function of the Higgs boson $p_{T}$ in a fiducial phase space defined by the leading(subleading) lepton $p_{T}>20(10) \mathrm{GeV},|\eta|<2.5$ $, m_{l l}>12 \mathrm{GeV}, p_{T}^{l l}>30 \mathrm{GeV}, m_{T}^{l l}>50 \mathrm{GeV}$ and $E_{T}^{\text {miss }}>0$. Experimentally the Higgs transverse momentum is defined by $\vec{p}_{T}^{H}=\vec{p}_{T}^{l l}+\vec{p}_{T}^{\text {miss }}$. In the figure Fig.3 we present the results and one can see that due to the low mass resolution (missing $p_{T}$ ) the unfolding matrix has large off diagonal elements.

\section{Conclusion}

Differential and integrated measurements of the fiducial cross sections using $H \rightarrow Z Z \rightarrow 4 l$, 

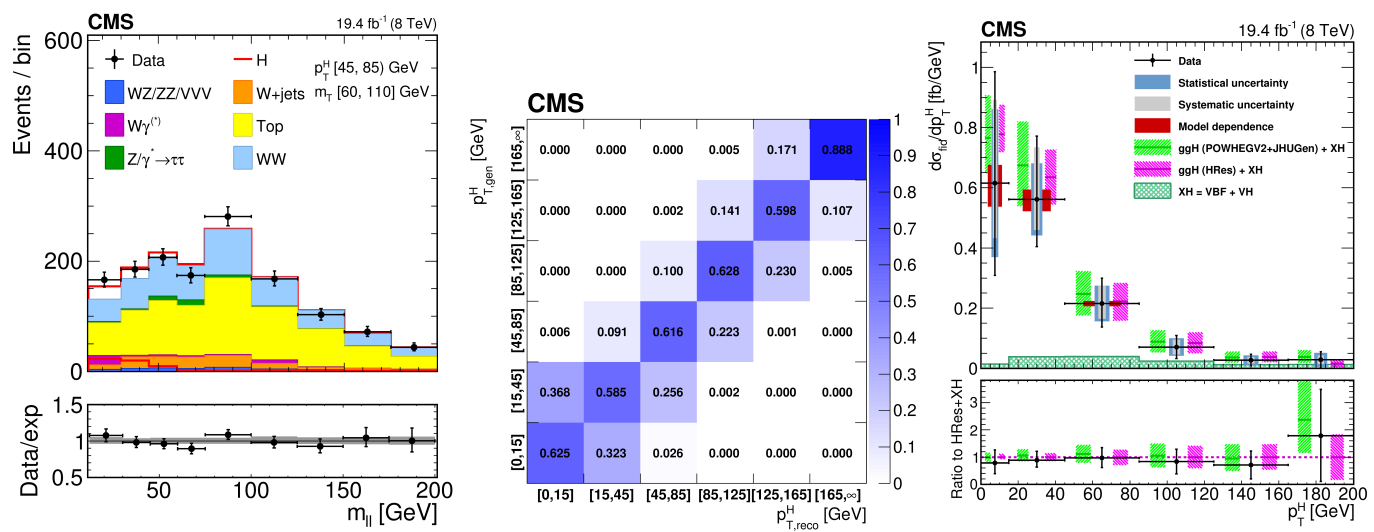

Figure 3: The dilepton mass distribution, the unfolding matrix and the Higgs differential cross section as a function of its $p_{T}$ after the unfolding

$H \rightarrow \gamma \gamma$ and $H \rightarrow W W \rightarrow 2 l 2 v$ decay channels were performed and compared to different theoretical predictions. Model dependence was estimated using a wide range of models and no significant deviations are observed in current datasets. In the future analysis should try to harmonise approaches to fiducial measurements according to the guidelines of the LHC Higgs cross section working group [6].

\section{References}

[1] CMS Collaboration, J. High Enery Phys. 04 (2016) 005

[2] CMS Collaboration, Eur. Phys. J. C 76 (2016) 13

[3] CMS Collaboration, Submitted to J. High Energy Phys. (arXiv:1606.01522)

[4] CMS Collaboration, CMS PAS HIG-15-004

[5] CMS Collaboration, CMS PAS HIG-16-020

[6] LHCHXSWG -YR4(2016), https://cds.cern.ch/record/2157092 